\title{
Abstract/Keywords
}

\section{Liquid Crystal Displays}

\section{Fabrication and Measurement of a Twisted Nematic Liquid Crystal Display}

\author{
Michael J. Ford ${ }^{1}$, Penny S. Hale, Joe G. Shapter, Nico H. Voelcker and Eric R. \\ Waclawik* \\ School of Chemistry, Physics and Earth Sciences (SoCPES), Flinders University of South \\ Australia, GPO Box 2100, Adelaide, 5001, AUSTRALIA; *cherw@flinders.edu.au \\ ${ }^{1}$ Institute for Nanoscale Technology, University of Technology, Sydney, \\ PO Box 123, Broadway, NSW 2007
}

This article describes a set of experiments designed to explore some of the basic properties of liquid crystals. The relationship between molecular shape, molecular order and macroscopic properties, such as optical birefringence, phase transitions and electrically controlled birefringence, as demonstrated by the electro-optic effect, are investigated. Students are encouraged to consider the importance of surface phenomena on these properties and how molecular order at a surface can be transferred throughout the bulk of such a highly associated fluid. Experiments were performed using either a sandwich cell or a twist cell containing the aligned nematic liquid crystal 4'-pentyl-4-cyano biphenyl. In the first part, students investigate the anisotropic nature of this liquid crystalline material using the wave-retarding properties of an aligned sample confined within a sandwich cell. In the second part, students manufacture a twisted nematic liquid crystal display and characterise the important elements required in a working display device based on electro-optical 
principals. The experiments are suitable as a second year laboratory and were performed by students in two three-hour sessions. The experiments could be adapted to a three-hour laboratory by omitting either the sandwich, or the twist cell section, without compromising the learning objectives of the lab.

Keywords: Liquid crystal, Liquid crystal display, Nematic, Twist cell, Birefringence, Electro-optic effect. 


\section{Lab Summary}

\section{Liquid Crystal Displays}

\section{Fabrication and Measurement of a Twisted Nematic Liquid Crystal Display}

Michael J. Ford, Penny S. Hale, Joe G. Shapter, Nico H. Voelcker and Eric R. Waclawik* School of Chemistry, Physics and Earth Sciences (SoCPES), Flinders University of South Australia, GPO Box 2100, Adelaide, 5001, AUSTRALIA; *cherw@flinders.edu.au

Examples of technologies that utilise the liquid crystalline phase of matter are not difficult to find. The liquid crystal display is the most common application, twisted nematic liquid crystal displays are commonly found in digital wristwatches, in microwave ovens, mobile phones and video recorders. Worldwide, sales of flatscreen liquid crystal displays have been projected to reach $8 \%$ of the 91.8 billiondollar video display market in 2002 (1). With this in mind, intense efforts by researchers in industry and in academia continue to be made into the synthesis of new liquid crystal compounds and the characterisation of their properties (2). Additionally, the liquid crystalline state of matter is being exploited for emerging technologies, such as the creation of nanostructured materials (3). Despite this, undergraduate chemistry students are still unlikely to encounter liquid crystals in the course of their experimental studies (4).

The experiment described herein was developed for a modern laboratory course on nanostructures, as part of the undergraduate degree of Bachelor of Science in Nanotechnology at Flinders University (5). The nanotechnology-degree has been in 
place for three years and has proven to be very successful, attracting large numbers of high calibre students. In the second year of their degree, students specialise in either of two streams (a) Nanostructures and Laser Devices, or (b) Biodevices. Where possible, experiments relevant to both streams (such as this liquid crystal laboratory) were designed for this course. The projects were designed to demonstrate the relationship between molecular order and the optical and dielectric properties of the liquid crystalline state and the application of these properties to create a working display device. The laboratory is suitable for second year undergraduates, particularly if they have some background in physical chemistry and possibly optics.

Molecules that possess a liquid crystal phase are typically elongated. The anisotropy in their shape causes them to form highly associated fluids or mesophases over certain temperature ranges (thermotropic liquid crystals) or in some solvents (lyotropic liquid crystals) (6). The high degree of order and the anisotropy in shape of liquid crystals can be observed via x-ray diffraction patterns and as an anisotropy in viscosity, electrical, optical or other properties. Upon cooling, many of these molecules pass through several liquid crystal phases, corresponding to different degrees of molecular order. Using equipment that is readily available to an undergraduate teaching laboratory, a laser diode, a polariser and a photodiode as a detector, we describe a simple and inexpensive experiment to directly measure the birefringence, or linear dichroism, of a nematic liquid crystal sample. The experiment can be extended to investigate the performance of a twisted nematic liquid crystal display.

\section{Overview of Procedures and Concepts Learned}

Experimental Procedures 
The students are expected to complete three projects over the course of two three-hour laboratory sessions. The first task is to manufacture an aligned nematic liquid crystal cell so they may measure (a) the optical birefringence of the liquid crystal sample and (b) the nematic-isotropic phase transition. The liquid crystal cell is constructed from two microscope slides each coated with an alignment layer of polyvinyl alcohol (PVA). The alignment direction is established by rubbing the polymer surface with a velvet cloth for 10 minutes. Thin mylar spacers are used to hold the cell apart, which is then filled with liquid crystal by capillary action. The cell is subsequently sealed at the edges using araldite. Properties of nematic liquid crystal 4'-pentyl-4-cyano biphenyl (or 5CB) were investigated here, although any compound or mixture that is nematic liquid crystalline at room temperature would be equally suitable (eg. MBBA) (7). The cell containing 5CB is birefringent, a consequence of homogeneous orientation of the $5 \mathrm{CB}$ molecules that come into contact with the aligned polymer molecules at the surface (8). This surface orientation is transferred throughout the ensemble of liquid crystal causing a different optical retardation of polarised light depending whether the light is plane-polarised perpendicular or parallel to the sample. This experiment therefore demonstrates nicely how surface nanostructure can influence the bulk properties of a material through self-organisation.

The sample birefringence measurements are quite simple and inexpensive to perform. A polariser, rotatable sample stage, a He:Ne laser, or laser-diode light source, a photodiode detector and an oscilloscope are all that is required. Using a polarised light source the experiment is effectively performed with a crossed polariser arrangement. The vertically polarised laser output passes through the centre of the polariser (often referred to as the analyser) arranged in a horizontal alignment, thus no 
signal is detected at the photodiode. The aligned nematic liquid crystal cell is then placed between the polarisers on the rotatable stage. Photodiode signal was digitised and stored on computer for different angles between the polarsiation vector of the incident beam and the net orientation of the liquid crystal using the analogue-todigital converter channel of a National Instruments NI-DAQ 6024E data acquisition board and software written in Labview 6.1. Although the results could just as easily have been read directly from the oscilloscope display, in a separate part of their course students had been given instruction in basic electronics and programming in Labview. This project intended to highlight the use of these previously acquired skills towards data analysis in their experimental coursework.

According to the molecular-statistical theory of the nematic phase developed by Maier and Saupe, the overall alignment of the 5CB sample is described by a single order-parameter, $S(6,8)$ according to,

$$
S=\frac{1}{2}\left\langle\cos ^{2} \theta-1\right\rangle
$$

where $\theta$ is the angle between the long-axis of the molecule and the preferred overall alignment direction of the liquid crystal sample (often called the director, $\boldsymbol{n}$ ). Rotation of the cell (and hence 5CB director) modulates the laser intensity at the photodiode, yielding results typical of those displayed in Figure 1. Two maxima and two minima appear for $360^{\circ}$ rotation corresponding to the 5CB-director being $45^{\circ}$ to, or aligned with the polarisation direction of the laser light. The birefringence of the nematic liquid crystal is given by the difference in refractive index measured when plane polarised light is parallel or perpendicular with respect to the director, 
$\Delta n=n_{/ /}-n_{\perp}$. A useful property of liquid crystals is that they have a large $\Delta n$, typically ranging between 0.05 to 0.5 over the infrared, near infrared, optical and ultraviolet regions of the spectrum (9). Any polarisation state can be produced by a combination of $\Delta n$ and sample thickness $d$. For monochromatic light of wavelength $\lambda$, the phase shift is given by

$$
\Delta \Phi=\frac{2 \pi}{\lambda} d\langle\Delta n\rangle
$$

The intensity of transmitted laser light through the sample depends upon this phase shift and $\varphi_{0}$, the angle between the polarisation vector of the incident beam and the orientation of the director, given by,

$I=I_{0} \sin ^{2}\left(2 \varphi_{0}\right) \sin ^{2}(\Delta \Phi / 2)$

where $I_{0}$ is the intensity of plane polarised light incident upon the cell (9). Students are asked to compare their results to a plot of $I=$ constant $x \sin ^{2}\left(2 \varphi_{0}\right)$, (represented by the solid line in Figure 1) and to calculated the thickness of sample required to create a quarter-wave plate out of 5CB material.

The second task for the students to perform was the measurement the nematic-toisotropic phase transition of 5CB using the same "crossed-polariser" arrangement. This experiment required temperature control of the liquid crystal sample. The liquid crystal sample cell was clipped to a metal block that was maintained at a precise temperature with a peltier. The block's temperature was monitored by a thermistor 
that controlled the operation of the peltier in a continuous feedback system. Using this apparatus, students measured the change in optical transmission through the sample and a crossed polariser as the temperature of the liquid crystal sample was increased above the nematic-isotropic transition temperature. As the sample temperature increases, the nematic-isotropic transition temperature is reached where the intermolecular forces that orient the 5CB molecules are overcome. At this critical temperature, all cooperative effects in the sample are lost and the liquid crystal spontaneously melts to form an isotropic liquid. This arrangement yielded a nematicisotropic transition temperature of $\sim 32{ }^{\circ} \mathrm{C}$ (the nematic-isotropic transition of $5 \mathrm{CB}$ is $\left.35^{\circ} \mathrm{C}\right)(10)$. The transition is shown in Figure 2. A useful adjunct to this experiment might be the measurement of liquid crystal order parameter as a function of temperature by means of its photometric absorbance. Such an experiment has been described before using a guest-dye molecule dissolved in a nematic liquid crystal host. The dye is aligned by the nematic host and hence a difference in absorptivity is observed when the long axis of the dye is oriented parallel to the polarised light source compared to a perpendicular orientation $(4,11)$.

In the second three-hour session, students manufactured a twisted nematic liquid crystal cell in order to demonstrate the electro-optic effect. The twisted nematic cells were constructed using InSnO conductive glass plates. The conductive sides of the conductive glass were identified using an ohm meter, before masking off a $4 \mathrm{~mm}$ section at the edge of each slide using scotch-tape. As previously, slides were coated with an alignment layer of PVA. The PVA was allowed to cure at room temperature for ten minutes, after which time the tape was peeled from the coated slides. The slides were placed in an oven at 60 degrees for 20 minutes to complete the curing 
process. The uncoated section of each conductive slide could be readily located. An alignment was established on the slides by rubbing with velvet cloth for 10 minutes as before. Care was taken to ensure that students establish the alignment direction of one slide perpendicular to the uncoated section while the alignment (rubbing) direction of the second slide was parallel. The twisted nematic liquid crystal display was constructed from the coated InSnO glass slides by placing the conducting side of one slide face-down towards the conductive side of the second slide upon which two thin $23 \mu \mathrm{m}$ strips of insulating mylar spacers had been laid. Two bulldog-clips were used to fasten the cell together before filling it with 5CB. Since the surface orientation at the coated slides was $\pi / 2$ with respect to each other, a twisted nematic liquid crystal cell was thereby created. Connection of an external circuit to the conductive sections of the glass completed the twisted nematic cell.

The optical response of this home-built liquid crystal display to an applied electromagnetic field was compared to the performance of a commercial twisted nematic liquid crystal display. When a potential difference is placed across certain compounds such as liquid crystal 5CB, the long-axis of the molecules align themselves with respect to the electromagnetic field. This effect is sometimes referred to as electrically controlled birefringence (ECB). An AC voltage output of a signal generator was used to control the birefringence in these experiments. The cell can be operated in a crossed polarisers arrangement, or between parallel polarisers. The intensity of transmitted light at the detector was monitored when a voltage was applied to the cell. Students first measured the threshold voltage at which their twisted nematic liquid crystal cell functioned. The threshold voltage for operation of the cell could then be compared to the commercial TNLCD. The transmission- 
voltage curve (TVC) for a home-built twisted nematic cell that has been placed between parallel polarisers is shown in Figure 3. The steepness of the TVC is an important factor for a twist cell defined by the steepness parameter $p(8)$, for crossed polarisers,

$p_{50}=U_{50} / U_{90}-1, p_{10}=U_{10} / U_{90}-1$

where $U_{10}, U_{50}$ and $U_{90}$ correspond to $10 \%, 50 \%$ and $90 \%$ optical transmission.

The speed with which the liquid crystal display responded to an applied voltage was also measured (see Figure 4). The rise time $\left(t_{\mathrm{r}}\right)$ decreases with decreasing applied voltage, as does the decay time $\left(t_{\text {decay }}\right)$. The bounce on the decay curves occurring due to backflow, where rotation of the director was also accompanied by movement of the liquid. This occurs for high driving voltages where $U>2 U_{\text {th }}$, where $U_{\text {th }}$ is the threshold voltage for reorientation (9). Invariably the response of the home-built device was slower than the commercial TNLCD. Students were required to speculate as to the reasons for the relatively slower response. The important factors being (1) the quality of the alignment polymer layer and (2) the thickness of the sample, a thicker sample resulting in a higher threshold voltage before switching of the device occurs and a slower response (represented by the steepness parameter $p$ ) to the applied electric field.

\section{Conclusions}

The experiment was usually performed by groups of three students. The groups were free to decide amongst themselves which tasks they wished to perform and the order 
with which the tasks were carried out. Responses to this set of experiments were positive. Students appeared to enjoy making a working display. This introduction to the liquid crystal state of matter and the connection between concepts learned in other topics in their course was appreciated. While the three experiments described herein (optical birefringence measurements, phase transition measurements and display performance measurements) were performed over two three hour sessions, each experiment could quite easily be adapted to a three-hour laboratory as an introduction to the properties of liquid crystals. In summary, students were given the opportunity to explore the relationship between molecular structure and intermolecular interactions that leads to properties observed at the macroscopic scale. The observed properties of the bulk material (electric and optical birefringence) were then used in construction of a useful technological device, a twisted nematic liquid crystal display.

\section{Acknowledgments}

The authors wish to acknowledge the Flinders University Faculty of Science and Engineering for its Financial support. We thank Peter Pallfy-Muhoray for his helpful suggestions on making an effective twisted nematic liquid crystal display.

\section{Supplemental Material}

Supplemental material for this article is available in this issue of JCE Online.

\section{Literature Cited}

1. Chin, S. Electronic Buyer’s News, Manhasset, 2002, 1315 (Jun. 3), 1. 
2. Depp, S. W.; Howard, W. E. Sci. Am. 1993, 268, 40.

3. Wilson, M.; Kannangara K.; Smith G.; Simmons, M.; Raguse, B. Nanotechnology. Basic Science and Emerging Technologies, UNSW Press, 2002.

4. For examples, see: Verbit, L. J. Chem. Educ. 1972, 49, 36. Dupré, D. B.; Chapoy, L. L. J. Chem. Educ. 1979, 56, 759. Patch, G.; Hope, G. A. J. Chem. Educ. 1985, 62, 454.

5. Shapter, J. G.; Ford, M. J.; Maddox, L. M.; Waclawik, E. R. IJEE. “in press”.

6. Chandresakar, S. Liquid Crystals, Cambridge University Press, 1977.

7. Liberko, C. A.; Shearer, S. J. Chem. Educ. 2000, 77, 1204.

8. Khoo, I-C., Liquid Crystals. Physical Properties and Nonlinear Optical Phenomena, John Wiley \& Sons, 1995.

9. Chigrinov, V. G., Liquid Crystal Devices: Physics and Applications, Artech House, 1999.

10. Waclawik, E. R.; Goh, M. C.;Donaldson, D. J., J. Chem. Phys. 1999, 110, 8098.

11. Demirbas, E.; Devonshire R. J. Chem Educ. 1996, 73, 586. 


\section{Figure Captions}

Figure 1: Transmitted light intensity through an aligned sample of 5CB placed between crossed polarisers as a function of director angle. Signal maxima are observed when the sample director is $45^{\circ}$ with respect to the polarisation axes of the laser and analyser. Signal minima are observed when the sample director is parallel to one of the polariser axes $\left(0^{\circ}\right.$ and $\left.90^{\circ}\right)$. The line drawn in the figure corresponds to the sine-squared distribution given by Equation 3, normalised to the maximum laser intensity.

Figure 2: Typical results for optical transmission versus temperature for a liquid crystal director aligned $45^{\circ}$ with respect to the laser and polariser.

Figure 3: Optical transmission of a home-built twisted nematic cell between parallel polarisers. Voltages corresponding to $10 \%, 50 \%$ and $90 \%$ transmission are marked giving steepness parameters $p_{10}=0.4$ and $p_{50}=0.5$.

Figure 4: Response time for the twist effect. The dark lines represent the decay and the rise oscillograms for $U_{90}$ for a home-built twisted nematic display placed between parallel polarisers (labelled a). The grey lines represent oscillograms for $U_{75}$ (labelled b). 
Figures

Figure 1

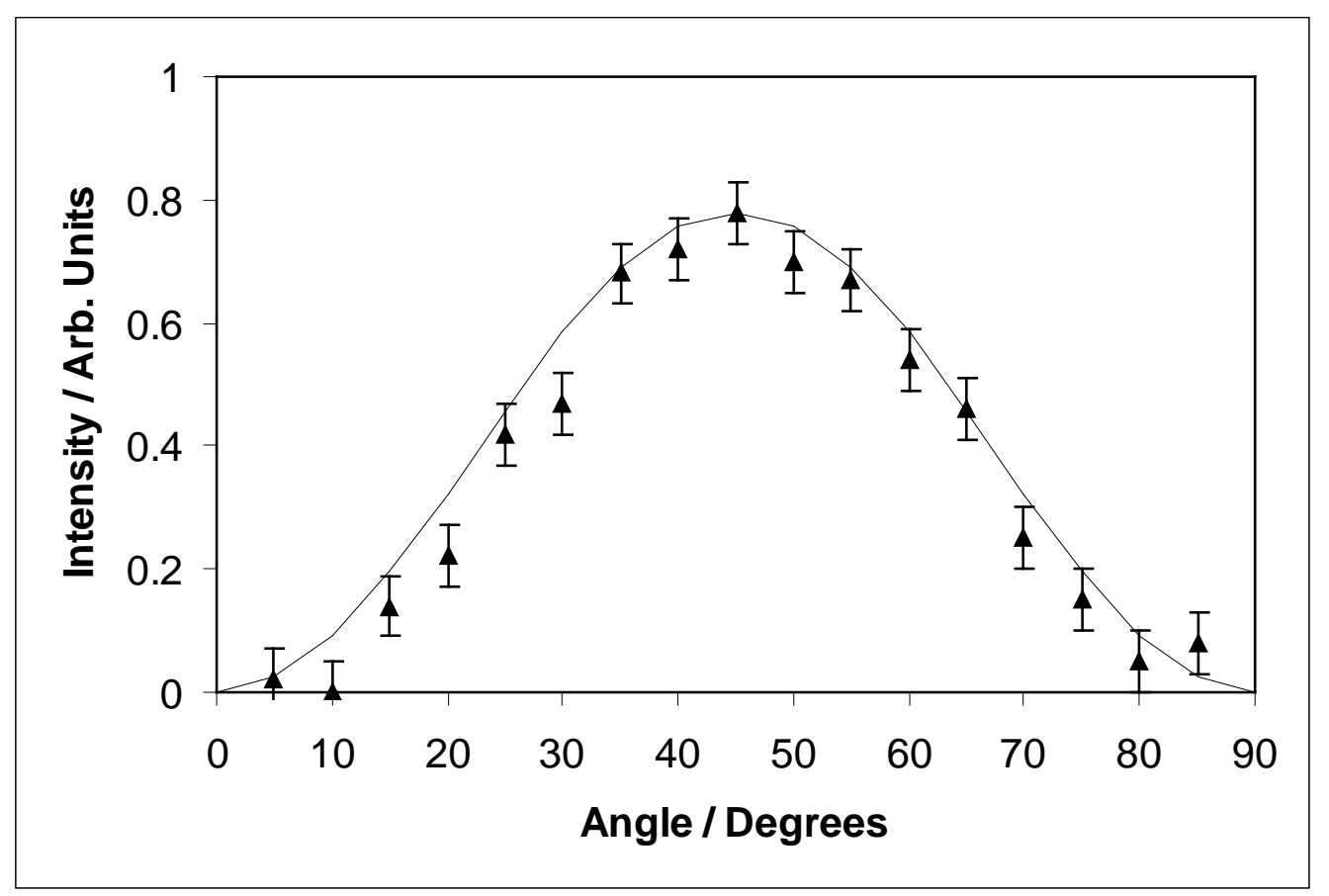


Figure 2

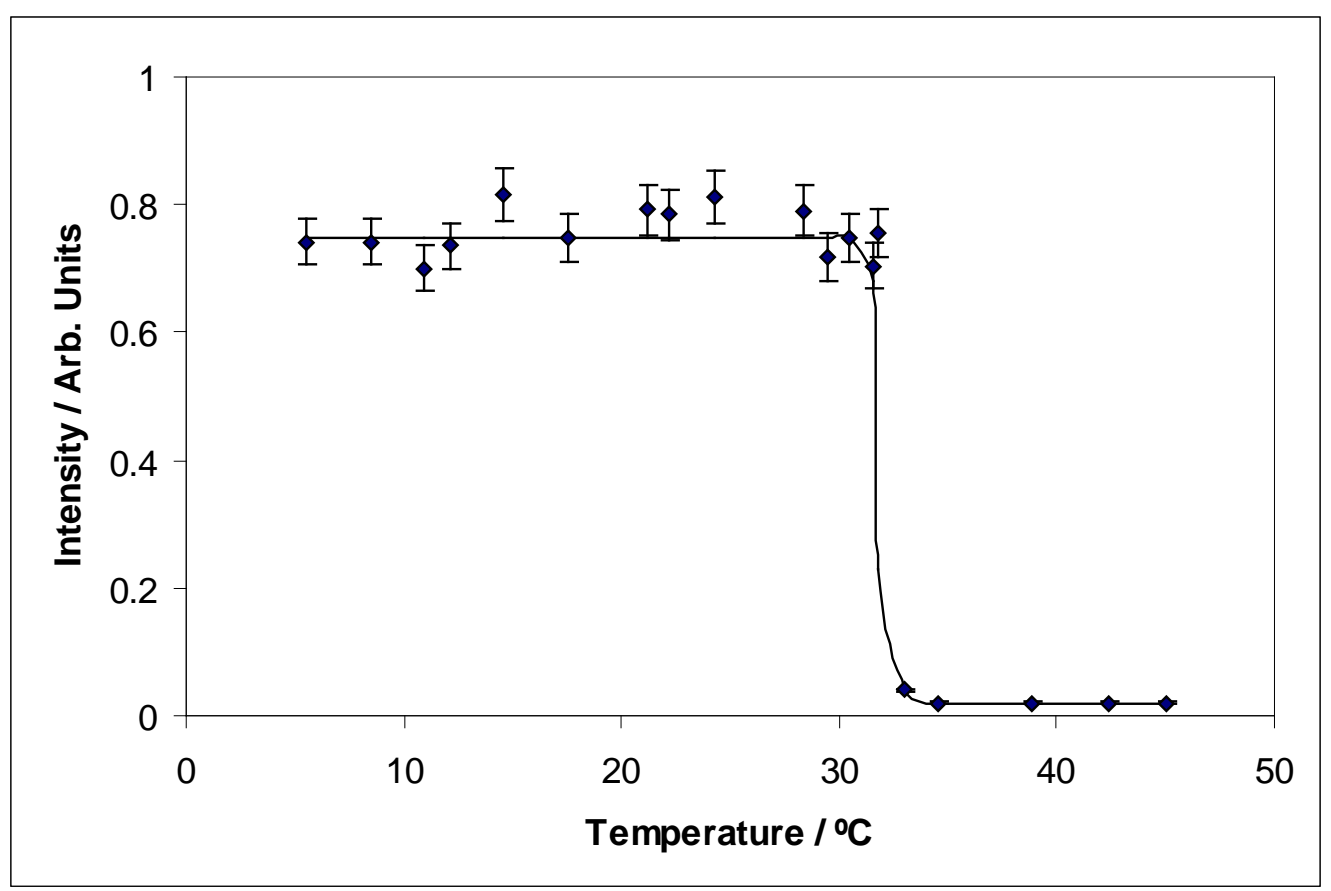


Figure 3

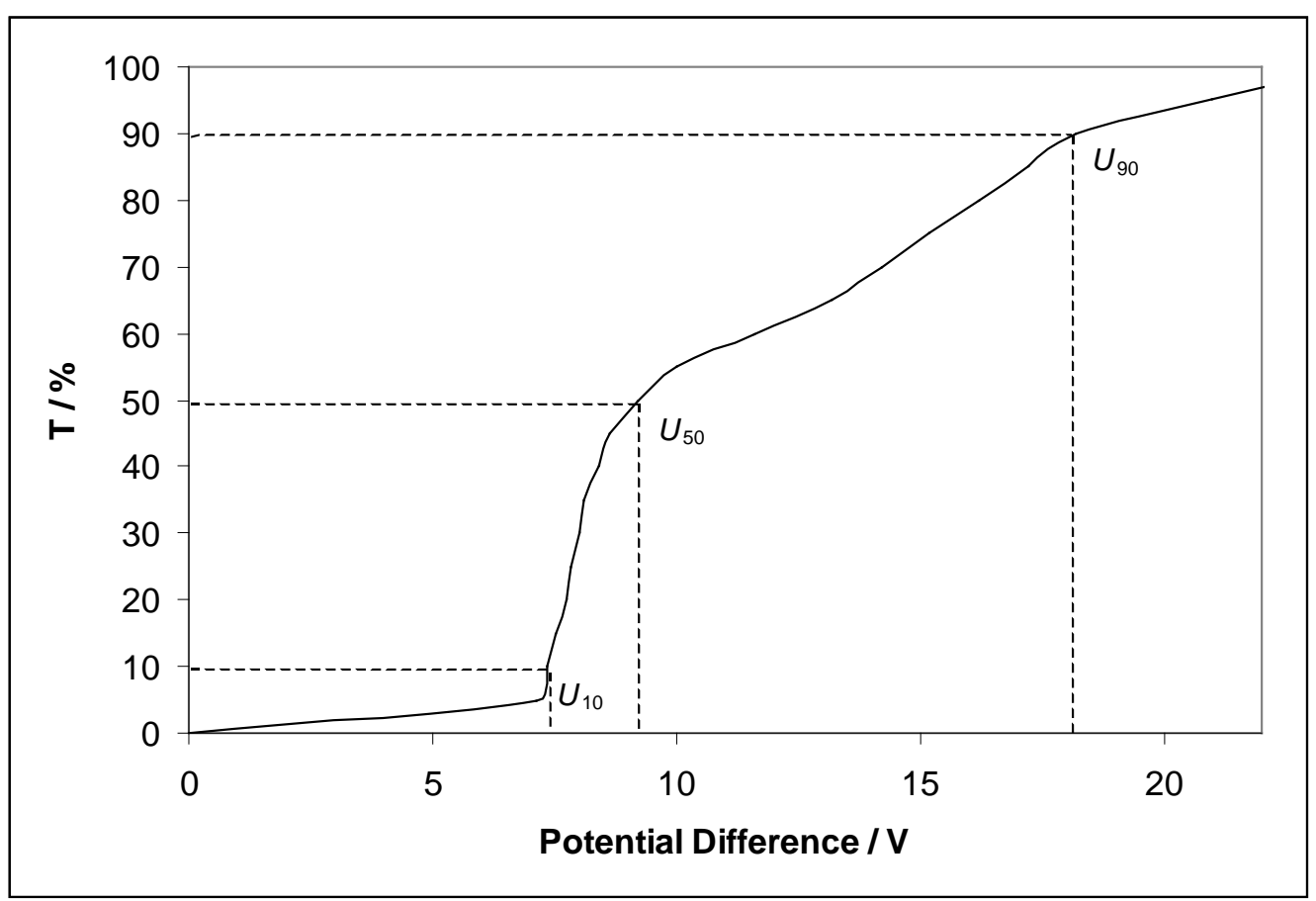


Figure 4

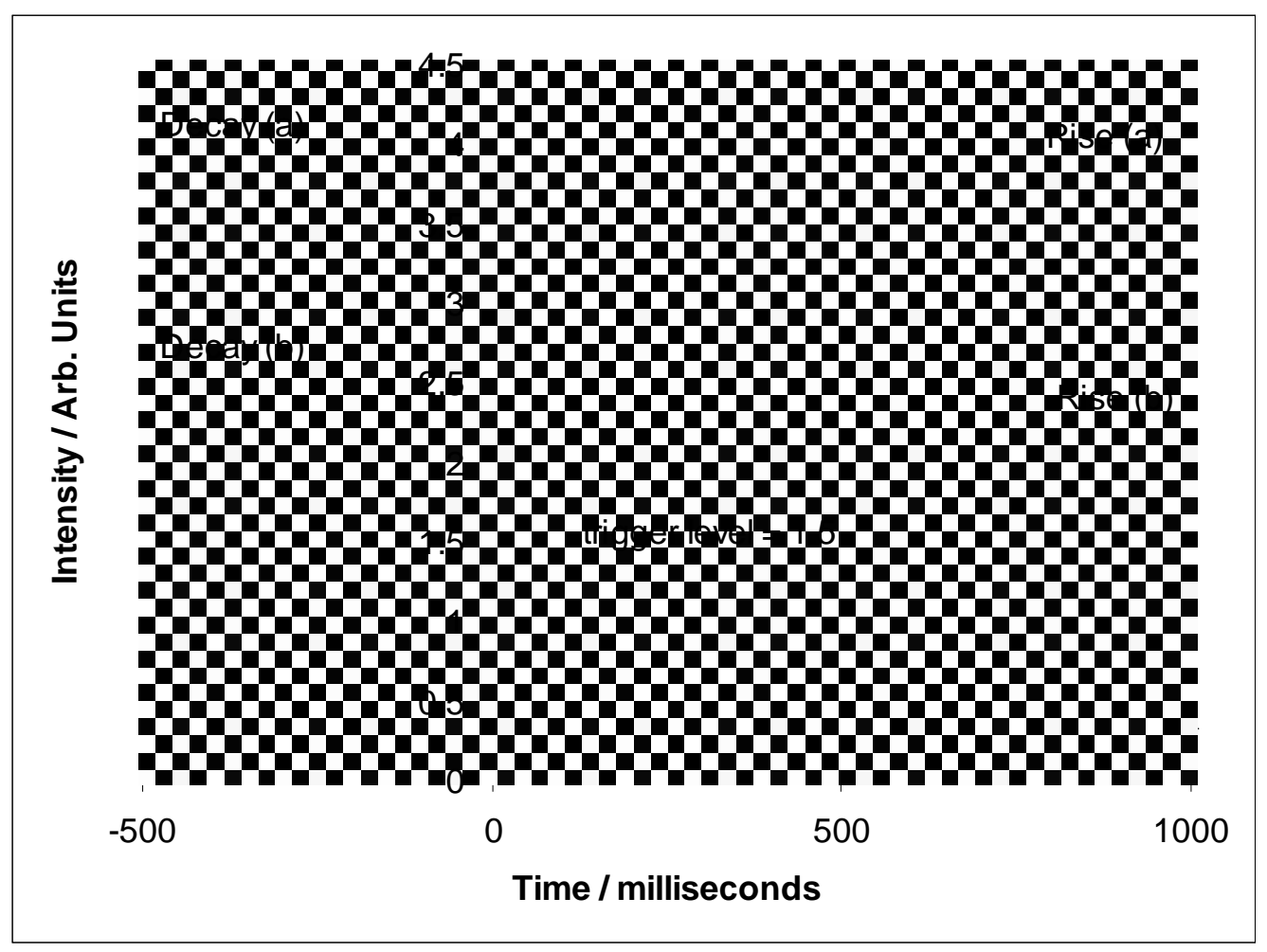

